\title{
Applying fuzzy multi-criteria decision method to evaluate key capabilities of Taiwan motion
} picture companies

\author{
Yaw-Chu Chen ${ }^{1} \quad$ Kuei-Lun Chang ${ }^{2}$ \\ ${ }^{1}$ Department of Advertising, Ming Chuan University \\ ${ }^{2}$ Graduate Institute of Industrial and Commerce Management, National Taipei University of Technology
}

\begin{abstract}
The purpose of this paper is to provide an algorithm for the motion picture companies to evaluate the key capabilities from firm viewpoint under fuzzy environment. The fundamental concepts we have adopted include the eigenvector method, fuzzy Delphi method, fuzzy set theory, and multi-criteria decision making method. With the concept of balanced scorecard and the interviewing managers of motion picture industry in Taiwan, we collect twenty three sub-criteria and construct the hierarchical structure for evaluating the motion picture company's key capabilities. The fuzzy Delphi method is integrated with the eigenvector method to form a set of pooled weights of the criteria. The concepts of triangular fuzzy number and linguistic variables are used to assess the preference ratings, including 'importance' and 'appropriateness', of linguistic variable. Through the hierarchy integration, we obtain the final scores of capabilities, and then use a revised Chang and Chen's ranking method for choosing the key capability.
\end{abstract}

Keywords : Balanced scorecard, Fuzzy multi-criteria decision making, Motion picture company, Cultural and creative industry, Key capability

\section{Introduction}

Recently, modern countries start to promote cultural and creative industry. Britain is the first country to propose the concept of creative industry and implement the policy. The UK government branded the cultural and creative industry as creative industry and defines it as comprising activities which have their origin in individual creativity, skills and talent, and which have the potential for wealth and job creation through generation and exploitation of intellectual property [9].

In 2002, Taiwan proposed 'Challenge 2008National development plan' to promote cultural and creative industry. Motion picture industry is included in this project [11]. In this paper, we define capability as the ability to make use of resources to perform some task or activity [12]. Key capabilities are valuable capabilities which help to generate high profit margins, and are the clear market winners in securing market share [13].

Kaplan and Norton [16] developed the concept of the balanced scorecard which is an approach to strategic and performance measurement and control. Balanced scorecard includes both financial and non-financial measures. Most non-financial measures are qualitative by nature and often depend on experts' judgment of approximation [12]. Such factors involve a great degree of linguistic deficiency [18]; in other words, we cannot elicit the complete, precise, and reliable knowledge from the experts. Due to the existing fuzziness in decision making as mentioned above, a fuzzy approach appears to be one of the feasible solutions for us to handle such difficult problems.

In this paper, an algorithm of motion picture company key capability evaluation is proposed on the basis of fuzzy set theory [25], paired comparison judgments of analytic hierarchy process [24] and fuzzy Delphi method [15]. The normal triangular fuzzy numbers [10] and the linguistic values are utilized to assess the preference ratings of linguistic variables [26]. Section 2 discusses sources of sub-criteria. Section 3 presents the method of this paper. Section 4 presents a Taiwan case study of motion picture company key capability evaluation, whereas Section 5 is the conclusion.

\section{Sources of sub-criteria}

Reviewing literatures $[1,2,3,4,8,14,16,17,19,20$, $21,22,23]$ and interviewing Taiwan motion picture industry officers, we collect twenty three sub-criteria. We use Likert seven point scales to evaluate the importance of sub-criteria. In this paper, the threshold level is $70 \%$, that is, the sub-criteria which 
the geometric mean values are less than 4.9 were removed. The evaluating sub-criteria are described below.

(1) Innovation and learning $\left({ }^{1} C_{1}\right)$ : \{employees' competency $\left({ }^{2} C_{1}\right)$,employees' productivity $\left({ }^{2} C_{2}\right)$, organizational culture $\left({ }^{2} C_{3}\right)$,employees' training

$\left({ }^{2} C_{4}\right)$,employees' satisfaction $\left({ }^{2} C_{5}\right)$, empowerment

$\left({ }^{2} C_{6}\right)$,adopt new technology $\left({ }^{2} C_{7}\right)$, number of suggestions $\left({ }^{2} C_{8}\right)$ \}.

(2) Internal business process $\left({ }^{1} C_{2}\right):$ new product $\left({ }^{2} C_{9}\right)$, cycle time $\left({ }^{2} C_{10}\right)$, data timeliness $\left.\left({ }^{2} C_{11}\right)\right\}$.

(3) Customer $\left({ }^{1} C_{3}\right):$ social responsibility $\left({ }^{2} C_{12}\right)$, brand reputation $\left({ }^{2} C_{13}\right)$, customer retention $\left({ }^{2} C_{14}\right)$, brand awareness $\left({ }^{2} C_{15}\right)$, customers' satisfaction $\left({ }^{2} C_{16}\right)$, customer relationship management $\left({ }^{2} C_{17}\right)$,product quality $\left({ }^{2} C_{18}\right)$, customer acquisition $\left({ }^{2} C_{19}\right)$ \}.

(4) Financial $\left({ }^{1} C_{4}\right)$ : \{new market $\left({ }^{2} C_{20}\right)$, cost per product $\left({ }^{2} C_{21}\right)$,profitability $\left({ }^{2} C_{22}\right)$,revenue growth $\left.\left({ }^{2} C_{23}\right)\right\}$.

\section{Method}

This section gives a fuzzy algorithm for motion picture company key capability evaluation as a background for further application. The triangular fuzzy number and linguistic variable are the two main concepts used in this paper to assess the preference ratings of linguistic variables, 'importance' and 'appropriateness'. The decision makers can employ an assumed weighting set $W=$ \{Very Low, Low, Medium, High, Very High\} to assess the relative importance of various criteria. And use the linguistic rating set $S=\{$ Very Poor, Poor, Fair, Good, Very Good $\}$ to evaluate the appropriateness of the alternatives versus various criteria. The membership functions of linguistic values in the weighting set $W$ and the linguistic rating set $S$ can be represented by approximate reasoning of triangular fuzzy numbers, as shown in Table 1[5].

Table 1. Membership functions for linguistic values

\begin{tabular}{ll}
\hline Linguistic values & Fuzzy numbers \\
\hline Very low (VL),Very poor (VP) & $(0,0,0.25)$ \\
Low (L), Poor (P) & $(0,0.25,0.5)$ \\
Medium (M), Fair (F) & $(0.25,0.5,0.75)$ \\
High (H), Good (G) & $(0.5,0.75,1)$ \\
Very high (VH), Very good (VG) & $(0.75,1,1)$ \\
\hline
\end{tabular}

A more general representation of multi-criteria decision making problem is introduced. Supposed there is a committee of $n$ decision makers $\left(D_{1}, D_{2}, \ldots\right.$, $D_{n}$ ) who are responsible for assessing the appropriateness of $m$ alternatives $\left(A_{1}, A_{2}, \ldots, A_{m}\right)$ under each of $k$ criteria $\left({ }^{h} C_{t}, t=1,2, \ldots, k, h=1,2\right.$; where $h=1$ means criteria and $h=2$ means sub-criteria) as well as the importance weight of the criteria. Let the $S_{i t j}(i=1,2, \ldots, m ; t=1,2, \ldots, k ; j=$ $1,2, \ldots, n)$ be the rating assigned to alternatives $A_{i}$ by decision maker $D_{j}$ under criterion ${ }^{h} C_{t}$.
An algorithm of the multi-person multi-criteria motion picture company key capability evaluation with fuzzy set approach can be expressed by the following steps :

Step 1. Construction of hierarchical structure

(1) Form a committee of decision makers, and then identify the evaluation criteria and alternative capabilities of motion picture company.

(2) Construct the hierarchical structure of motion picture company key capability evaluation through the concept of balanced scorecard.

Step 2. Evaluation of the importance weight of each criterion

(3) Use fuzzy Delphi method to determine the fuzzy number of pooled weight of each criterion.

Step 3. Construction of linguistic scales for linguistic variables

(4) Choose the appropriate preference ratings for the importance weight of the evaluation criterion.

(5) Select the appropriateness ratings for alternatives under sub-criteria.

Step 4. Aggregation of fuzzy appropriateness indices

(6) Aggregate the weight of sub-criterion to get the aggregated weight ${ }^{2} W_{t}$.

(7) Pool the decision makers' opinions to get the aggregated fuzzy rating $S_{i t}$ of alternative $A_{i}$ under each sub-criterion ${ }^{2} C_{t}$.

(8) Aggregate $S_{i t}$ and ${ }^{2} W_{t}$ with respect to each sub-criterion to obtain the fuzzy appropriateness indices $R_{i}$ for all alternatives.

Step 5. Computation of fuzzy overall evaluation

(9) Aggregate polled weight $\left({ }^{1} W_{t}\right)$ of criteria with fuzzy appropriateness indices $\left(R_{i}\right)$ to obtain the fuzzy overall evaluation $\left(F_{i}\right)$ of each alternative.

Step 6. Defuzzification of fuzzy overall evaluation

(10)Calculate the ranking value $U_{T}\left(F_{i}\right)$ by defuzzifying the fuzzy overall evaluation through ranking method.

Step 7. Analysis and decision

(11)Choose the capability of motion picture company with the maximal ranking value.

\section{An application}

The case company is Central Motion Picture Corporation (CMPC). Founded in 1954, CMPC is the largest, most comprehensive and historically the oldest movie production company in Taiwan.

Step 1.

A committee of four decision makers, $D_{1}, D_{2}$, $D_{3}$ and $D_{4}$, has been formed to determine the key capability of motion picture company. They are deputy manager of sales department, manager of finance department, deputy manger of edit department and manager of international business development department.

There are six capabilities of motion picture company in the case study. The six capabilities are 
script selection, films production, films investment, copyright selling, advertisement selling and location renting.

\section{Step 2.}

There are some easy ways to obtain a good approximation of the priorities. One of the best ways is the geometric mean [24]. By normalizing of geometric mean of the rows and using fuzzy Delphi concept [15], we can derive the final weights for the criteria of level 2.

Due to the property of fuzzier in criteria than in sub-criteria, we use triangular fuzzy number to aggregate the decision makers' assessments and geometric mean to obtain fuzzy weight ${ }^{1} W_{t}$. Define

${ }^{1} W_{t}=\left(e_{t}, f_{t}, g_{t}\right)$

where ${ }^{1} W_{t}$ is the fuzzy weight under criterion $t$, $e_{t}=\min _{j}\left\{W_{j t}\right\}, \quad g_{t}=\max _{j}\left\{W_{j t}\right\}, f_{t}=\left(\prod_{j=1}^{n}{ }^{1} W_{j t}\right)^{1 / n}$, $t=1,2, \ldots, 4, \quad j$ are decision makers.

By using equation (1), we obtain the importance of the criteria as shown in Table 2.

Table 2. The importance of the criteria

\begin{tabular}{ll}
\hline Criteria & Weight \\
${ }^{1} C_{1}$ & ${ }^{1} W_{1}=(0.1352,0.2306,0.3205)$ \\
${ }^{1} C_{2}$ & ${ }^{1} W_{2}=(0.0879,0.2019,0.3126)$ \\
${ }^{1} C_{3}$ & ${ }^{1} W_{3}=(0.1045,0.1838,0.2481)$ \\
${ }^{1} C_{4}$ & ${ }^{1} W_{4}=(0.1372,0.2853,0.6725)$ \\
\hline
\end{tabular}

Step 3.

The decision makers can use the importance weighting set $W$ and appropriateness ratings set $S$ described in Table 1, to evaluate the importance weight ${ }^{2} W_{t}$, and the appropriateness ratings $S_{i t}$ for candidates under sub-criteria, respectively.

\section{Step 4.}

We will use the mean operator to aggregate the decision makers assessments. Let $\oplus$ and $\otimes$ be fuzzy addition and multiplication operators respectively. Define

$$
\begin{aligned}
& S_{i t}=\left(\frac{1}{n}\right) \otimes\left(S_{i t 1} \oplus S_{i t 2} \oplus \cdots \oplus S_{i t j} \oplus \cdots \oplus S_{m k n}\right) \\
& { }^{2} W_{t}=\left(\frac{1}{n}\right) \otimes\left({ }^{2} W_{t 1} \oplus^{2} W_{t 2} \oplus \cdots \oplus^{2} W_{t j} \oplus \cdots \oplus^{2} W_{k n}\right)
\end{aligned}
$$

where $S_{i t}$ is the average fuzzy appropriateness rating of alternative $A_{i}$ under sub-criterion ${ }^{2} C_{t}$ and ${ }^{2} W_{t}$ is average importance weight of sub-criterion ${ }^{2} C_{t}$. Thus the fuzzy appropriateness index $R_{i}$ of the alternative can be obtained by aggregating $S_{i t}$ and ${ }^{2} W_{t}$ denoted as

$R_{i}=\left(\frac{1}{k}\right) \otimes\left[\left(S_{i 1} \otimes^{2} W_{1}\right) \oplus\left(S_{i 2} \otimes^{2} W_{2}\right) \oplus \cdots \oplus\left(S_{i t} \otimes^{2} W_{t}\right) \oplus \cdots \oplus\left(S_{i k} \otimes^{2} W_{k}\right)_{-}^{-}\right.$

$$
\cong\left(Y_{i}, Q_{i}, Z_{i}\right)
$$

\section{Step 5.}

The fuzzy overall evaluation of six alternatives as shown in Table 3 can be obtain by multiplying $R_{i}$ and ${ }^{1} W_{t}$ denoted as

$F_{i} \cong R_{i} \otimes{ }^{1} W_{t} \cong\left(Y_{i}^{\prime}, Q_{i}{ }^{\prime}, Z_{i}{ }^{\prime}\right)$,

where $Y_{i}^{\prime}=Y_{i} \times e_{t}, Q_{i}^{\prime}=Q_{i} \times f_{t}, Z_{i}^{\prime}=Z_{i} \times g_{t}$;

$R_{i}$ and ${ }^{1} W_{t}$ can be obtain by using equation (4) and equation (1) respectively.

Table 3. The fuzzy overall evaluation of six alternatives

\begin{tabular}{ll}
\hline Alternatives & Overall evaluation \\
\hline$A_{1}$ & $F_{1} \cong 0.0607,0.3250,0.9544$ \\
$A_{2}$ & $F_{2} \cong 0.0607,0.3219,0.9548$ \\
$A_{3}$ & $F_{3} \cong 0.0767,0.3716,1.0237$ \\
$A_{4}$ & $F_{4} \cong 0.0496,0.2963,0.8992$ \\
$A_{5}$ & $F_{5} \cong 0.0178,0.2149,0.7804$ \\
$A_{6}$ & $F_{6} \cong 0.0509,0.2943,0.8826$ \\
\hline
\end{tabular}

Step 6.

Chen and Hwang [6] made distinctions into four categories between fuzzy ranking methods and fuzzy multiple attribute decision-making methods, left and right score method. According to Chen [7] which revised the Chang and Chen's ranking method [5], the ranking values $U_{T}\left(F_{i}\right)$ can be approximately obtained by

$$
\begin{aligned}
& U_{T}\left(F_{i}\right) \cong \hat{a}\left(Z^{\prime}{ }_{i}-x_{1}\right)\left(x_{2}-x_{1}-Q_{i}^{\prime}+Z_{i}^{\prime}\right) \\
& +(1-\hat{a}) \times\left[1-\left(x_{2}-Y_{i}^{\prime}\right)\left(x_{2}-x_{1}+Q_{i}^{\prime}-Y_{i}^{\prime}\right)\right\rfloor
\end{aligned}
$$

for $i \quad 1,2, \ldots, m$;

where $\hat{a}$ is the index of optimism in multi-person decision making proposed by Chen [7].

$$
\begin{aligned}
& x_{1}=\min \left\{Y_{1}^{\prime}, Y_{2}^{\prime}, \ldots, Y_{m}^{\prime}\right\}, \\
& x_{2}=\max \left\{Z^{\prime}, Z_{2}^{\prime}, \ldots, Z^{\prime}{ }_{m}\right\}, \\
& \text { By using equation } 6 \text {, we obtain the ranking }
\end{aligned}
$$
value of $F_{i}$ as shown in Table 4.

Table 4. The ranking values of the fuzzy overall evaluation for six alternatives

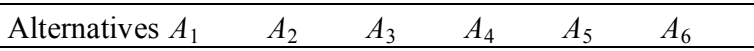
\begin{tabular}{lllllll}
\hline$U_{T}\left(F_{i}\right)$ & 0.4338 & 0.4324 & 0.4661 & 0.4112 & 0.3503 & 0.4076 \\
\hline
\end{tabular} Step 7.

Therefore, it is obvious that the key capability of motion picture company is $A_{3}$ i.e., films investment.

\section{Conclusion}

In this paper, a decision algorithm based on the fuzzy set theory is proposed to solve the motion picture company key capability evaluation problem. Through the concept of balanced scorecard, the hierarchical structure of motion picture company key capability evaluation on the basis of fuzzy set theory 
is constructed. The weights of four criteria are determined by analytic hierarchy process based on pairwise comparison. The fuzzy overall evaluation of each alternative comes from fuzzy multi-criteria decision making method through hierarchy aggregation. By using the revised Chang and Chen's [5] method proposed in this paper, we can determine the rank of fuzzy overall evaluation for six alternatives to determine the key capability of motion picture company.

\section{References}

[1]M. Anand, B. S. Sahay, S. Saha, Balanced scorecard in Indian companies, VIKALPA 30(2) (2005) 11-25.

[2]R. D. Banker, H. Chang, S. N. Janakiraman, C. Konstans, A balanced scorecard analysis of performance metrics, European Journal of Operational Research 154 (2004) 423-436.

[3]W. G. Bremser, Q. B. Chung, A framework for performance measurement in the e-business environment, Electronic Commerce Research and Applications 4 (2005) 395-412.

[4]D. Chand, G. Hachey, J. Hunton, V. Owhoso, S. Vasudevan, A balanced scorecard based framework for assessing the strategic impacts of ERP systems, Computers in Industry 56 (2005) 558-572.

[5]P. L. Chang, Y. C. Chen, A fuzzy multi-criteria decision making method for technology transfer strategy selection in biotechnology, Fuzzy Sets and Systems 63 (1994) 131-139.

[6]S. J. Chen, C. L. Hwang, Fuzzy Multiple Attribute Decision-making, Methods and Applications, Springer, Heidelberg, 1993.

[7]Y. C. Chen, An application of fuzzy set theory to the external performance evaluation of distribution centers in logistics, Soft Computing 6 (2002) 64-70.

[8]S. Davis, T. Albright, An investigation of the effect of balanced scorecard implementation on financial performance, Management Accounting Research 15 (2004) 135-153.

[9]Department of Culture, Media and Sport, The Creative Industries Mapping Report, HMSO, London, 1998.

[10]D. Dubois, H. Prade, Operating on fuzzy numbers, International Journal of Systems Science 9 (1978) 613-629.

[11]Executive Yuan, Challenge 2008 - National Development Plan, Council for economic planning and development, Taiwan, 2002.

[12]K. Hafeez, Y. B. Zhang, N. Malak, Determining key capabilities of a firm using analytic hierarchy process, International Journal of Production Economics 76 (2002) 39-51.
[13]K. Hafeez, Y. B. Zhang, N. Malak, Core competence for sustainable competitive advantage: A structured methodology for identifying core competence, IEEE Transactions on Engineering Management 49(1) (2002) 28-35.

[14]C. Hastings, Discussion of performance measures in public service broadcasting, Aslib Proceedings: New Information Perspectives 56(5) (2004) 301-307.

[15]T. H. Hsu, The fuzzy Delphi analytic hierarchy process, International Journal of Fuzzy Systems 4 (1) (1998) 59-72.

[16]R. S. Kaplan, D. P. Norton, The balanced scorecard-Measures that drive performance, Harvard Business Review 70(1) (1992) 71-79.

[17]R. S. Kaplan, D. P. Norton, Using the balanced scorecard as a strategic management system, Harvard Business Review 74(1) (1996) 75-85.

[18]G. J. Klir, Z. Wang, D. Harmanec, Constructing fuzzy measures in expert systems, Fuzzy Sets and Systems 92 (2) (1997) 251-264.

[19]E. K. Laitinen, Microeconomic analysis of the balanced scorecard: A case of Nokia Corporation, International Journal of Productivity and Performance Management 54(5/6) (2005) 325-339.

[20]J. Michalska, The usage of the balanced scorecard for the estimation of the enterprise's effectiveness, Journal of Materials Processing Technology 162-163 (2005) 751-758.

[21]K. Milis, R. Mercken, The use of the balanced scorecard for the evaluation of information and communication technology projects, International Journal of Project Management 22 (2004) 87-97.

[22]A. Papalexandris, G. Ioannou, G. P. Prastacos, Implementing the balanced scorecard in Greece: A software firm's experience, Long Range Planning 37 (2004) 351-366.

[23]A. Papalexandris, G. Ioannou, G. Prastacos, K. E. Soderquist, An integrated methodology for putting the balanced scorecard into action, European Management Journal 23(2) (2005) 214-227.

[24]T. L. Saaty, The Analytic Hierarchy Process, McGraw Hill, New York, 1980.

[25]L. A. Zadeh, Fuzzy sets, Information and Control 8 (1965) 338-353.

[26]L. A. Zadeh, The concept of a linguistic variable and its application to approximate reasoning, Information Sciences 8 (I) (1975) 199-249. 